Kompass

Autoimmun

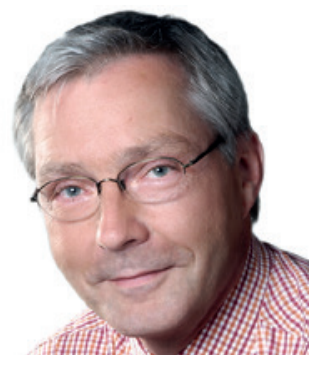

\title{
Einsatz von Secukinumab bei Patienten mit Psoriasis und Psoriasis-Arthritis - Ergebnisse einer nichtinterventionellen Studie unter Praxisbedingungen (SERENA-Studie)
}

Michael Sticherling

Universitätsklinikum Erlangen, Hautklinik, Erlangen, Deutschland

Abstract aus Kiltz U, Sfikakis PP, Gaffney K, et al.: Secukinumab use in patients with moderate to severe psoriasis, psoriatic arthritis and ankylosing spondylitis in real-world setting in Europe: baseline data from SERENA study. Adv Ther 2020;37:2865-2883.

\author{
Keywords \\ Ankylosing spondylitis · Biologics · Observational · Psoriasis . \\ Psoriatic arthritis · Real-world · Rheumatology · Safety · \\ Secukinumab
}

\begin{abstract}
Introduction: Secukinumab, a fully human monoclonal antibody that directly inhibits interleukin-17A, has demonstrated robust efficacy in the treatment of moderate to severe psoriasis (PsO), psoriatic arthritis (PsA) and ankylosing spondylitis (AS), with a rapid onset of action, sustained long-term clinical responses and a consistently favourable safety profile across phase 3 trials. Here, we report the clinical data at enrolment from SERENA, designed to investigate the real-world use of secukinumab across all three indications.
\end{abstract}

Methods: SERENA is an ongoing, longitudinal, observational study conducted at 438 sites across Europe in patients with moderate to severe plaque $\mathrm{PsO}$, active $\mathrm{PsA}$ or active AS. Patients should have received at least 16 weeks of secukinumab treatment before enrolment in the study.
Results: Overall 2800 patients were included in the safety set; patients with PsA ( $N=541)$ were older than patients with $\mathrm{PsO}$ $(N=1799)$ and patients with AS $(N=460)$; patients with PsO had a higher mean body weight than patients with PsA and patients with AS; and patients with PsO and patients with AS were predominantly male. Time since diagnosis was longer in patients with $\mathrm{PsO}$ compared with patients with PsA and patients with AS, and about $40 \%$ of patients were either current or former smokers. The proportion of obese patients (body mass index $\geq 30 \mathrm{~kg} / \mathrm{m}^{2}$ ) was similar across indications. Patients were treated with secukinumab for a mean duration of 1 year prior to enrolment (range 0.89-1.04). The percentages of patients with prior biologics exposure were 31.5\% PsO, 59.7\% PsA and 55\% AS. The percentages of patients prescribed secukinumab monotherapy were $75 \%(n=1349)$ in $\mathrm{PsO}, 48.2 \%(n=261)$ in PsA and $48.9 \%(n=225)$ in AS groups.

Conclusion: Baseline demographics of the study population are consistent with existing literature. This large observational study across all secukinumab indications will provide valuable information on the long-term effectiveness and safety of secukinumab in the real-world setting. 


\section{Transfer in die Praxis}

\section{Hintergrund}

Die Schuppenflechte gehört zu den häufigsten chronisch-entzündlichen Hauterkrankungen des Menschen. Bei etwa 30\% der Betroffenen tritt nach etwa 10 Jahren Verlauf der Hauterkrankung eine entzündliche Beteiligung von Enthesen und Gelenken auf, die zu den Spondylarthritiden gehören und unter dem Sammelbegriff Psoriasis-Arthritis zusammengefasst werden. Zur umfassenden Betreuung der Betroffenen ist daher eine gute interdisziplinäre Zusammenarbeit zwischen Dermatologen und Rheumatologen erforderlich.

Die entzündungshemmenden Biologika, vornehmlich monoklonale Anti-Zytokin-Antikörper, haben in den letzten Jahren das therapeutische Vorgehen sowohl der Haut- als auch der Gelenksmanifestationen grundsätzlich verbessert. Daten aus kontrollierten klinischen Studien sind dabei aufgrund ihrer Ein- und Ausschlusskriterien nur bedingt repräsentativ für die Patienten, die in der klinischen Praxis versorgt werden. Daher sind Untersuchungen unter diesen Bedingungen erforderlich, um das therapeutische Ansprechen auch vor dem Hintergrund vorbestehender komplexerer Begleiterkrankungen und Begleitmedikation zu evaluieren.

\section{Studienergebnisse}

Die SERENA-Studie ist eine laufende, longitudinale Beobachtungsstudie, die Patienten mit moderater bis schwerer Plaque-Psoriasis (PsO), aktiver Psoriasis-Arthritis (PsA) oder aktiver ankylosierender Spondylitis (AS) von 438 europäischen Zentren in 19 Ländern einschließt. Die betroffenen Patienten müssen über mindestens 16 Wochen zuvor Secukinumab angewendet haben. Die Studie ist angelegt für eine Dauer von 5 Jahren mit dokumentierten Patientenvisiten alle 6 Monate. Das Ende der Studie ist geplant, wenn entweder alle eingeschlossenen Patienten eine mindestens 2-jährige Nachverfolgung absolviert haben oder wenn die 5-Jahres-Visite bei etwa 1000 Patienten dokumentiert worden ist. Ausschlusskriterium sind schwere medizinische oder psychiatrische Zustände, die eine Beteiligung an der Studie für mindestens 2 Jahre beeinträchtigten. Primäres Studienziel ist die Erfassung der langfristigen Beibehaltung der Secukinumab-Therapie in täglicher Routineanwendung bei den genannten 3 Erkrankungen sowie die Erkennung von Faktoren, die diesen Verbleib beeinflussen. Die Datenerhebung der primären und weiteren Zielparameter erfolgte prospektiv, die zur Effektivität und Sicherheit jedoch auch retrospektiv seit Beginn der Secukinumab-Therapie. Krankheitsspezifische Parameter zur Erfassung der Effektivität und Lebensqualitätsparameter wurden bei den 3 Erkrankungen angewendet, darunter (1) für die Psoriasis der Psoriasis Area and Severity Index (PASI), die betroffene Körperoberfläche (body surface area (BSA)), der Physician's Global Assessment (PGA)-Score sowie das Ausmaß der Nagelbeteiligung, (2) für die PsA neben den PsO-Parametern der Total Joint Count (TJS, 78 Gelenke), der Leeds Enthesitis Index (LEI), eine visuelle Analogskala (VAS) für den Gelenkschmerz und (3) für die AS unter anderem der Bath Ankylosing Spondylitis Disease Activity Index (BASDAI), der Maastricht
Ankylosing Spondylitis Enthesitis Score (MASES), das C-reaktive Protein (CRP), Magnetresonanztomografie (MRT) und Röntgenuntersuchungen.

Insgesamt sind zum Zeitpunkt der Auswertung 2932 Patienten eingeschlossen worden, 2800 davon zur Medikamentensicherheit. Die größte Gruppe mit 1799 Patienten umfasste PsO-Patienten, 541 mit PsA und 460 mit AS. Die PsA-Patienten waren insgesamt älter als die PsO- und AS-Patienten mit einer Geschlechterverteilung von 67\%, 44\% und 59\% Männern bei PsO, PsA bzw. AS. Der Body Mass Index (BMI) war mit etwa 28 in allen 3 Gruppen gleichermaßen erhöht, das Intervall zwischen Erstmanifestation und Erstdiagnose bei PsO-Patienten aber deutlich länger als bei PsA und AS. Die Patienten der 3 Gruppen hatten vor Studieneinschluss zu einem hohen Prozentsatz von 30-60\% mindestens 1 anderes Biologikum angewendet, wobei 75\% der Patienten mit PsO, 48\% mit PsA und 49\% mit AS Secukinumab als Monotherapie erhielten. Der mittlere PASI vor Secukinumab-Therapie betrug 20 in der PsO- und 7,6 in der PsA-Gruppe, bei Einschluss in die Studie bei PsO 3 und 2,0 bei PsA. $55 \%$ der PsA-Patienten hatten eine mittlere Zahl geschwollener und schmerzender Gelenke von 6,4 bzw. 3,3. 22\% der PsO-Patienten ohne PsA, 32\% mit PsO und PsA sowie 18\% der PsA-Patienten zeigten eine Nagelbeteiligung. Auch bei den AS-Patienten lagen die spezifischen Krankheitsscores vor Therapie höher als bei Studieneinschluss. Insgesamt zeigten 26\% eine Enthesitis, 58\% jedoch eine normale CRP-Konzentration.

Die Rate von Unverträglichkeitsreaktionen, insbesondere solche von speziellem Interesse, war sehr niedrig mit einer Infektionsrate von 3,3\%. Diese Zahl war in der PsO-Gruppe mit 4\% höher als bei PsA oder AS. 2 Fälle mit entzündlichen Darmerkrankungen wurden in den 2 rheumatologischen Gruppen berichtet. Ansonsten fanden sich keine unerwarteten Sicherheitsaspekte.

\section{Kommentar}

Die Studie untersuchte die Effektivität und Verträglichkeit von Secukinumab in der täglichen Praxis bei moderater bis schwerer Plaque-Psoriasis, aktiver PsA und AS, vor allem aber die Therapietreue über die Zeit. Es konnten in dieser Studie Patienten eingeschlossen werden, die Begleiterkrankungen oder Begleitmedikation haben, die in einer kontrollierten randomisierten Studie zu einem Ausschluss geführt hätten. Damit geben nichtinterventionelle Studien zusätzliche Hinweise hinsichtlich der Effektivität und Verträglichkeit von therapeutischen Wirkstoffen im täglichen Leben. Auch die langfristige Beibehaltung von Secukinumab ist unter täglichen Lebensbedingungen erfasst worden. Das Diagnoseintervall war bei dieser Studie mit 9,3 Jahren deutlich höher als in publizierten kontrollierten Studien, wie auch die Krankheitsaktivität bei Studieneinschluss insgesamt niedriger war als in publizierten Phase3-Studien. Im Gegensatz zu Phase-3-Studien hatten die Patienten mehrere Biologika in der Vortherapie, ein Drittel der Patienten sogar mehr als 3. Dabei war ein Wirksamkeitsverlust der häufigste Anlass zum Absetzen der Medikation. Wesentliche Sicherheitsrisi- 
ken wurden in dieser Studie nicht berichtet, möglicherweise jedoch auch wegen der retrospektiven Erfassung im ersten Teil. Auch sind angesichts des Erfassungszeitraumes von bis zu 6 Jahren die zurückliegende Medikation und Unverträglichkeitsereignisse möglicherweise zu wenig oder nicht berichtet. Als Beobachtungsstudie finden sich keine Kontrollgruppe, zum Teil inkomplette Datensätze sowie ein Selektionsbias angesichts der obligaten Einleitung von Secukinumab vor Einschluss in die Studie. Auch die Beteiligung von 19 europäischen Staaten mit einem deutlichen Ungleichgewicht der Patientenzahlen und unterschiedlicher Krankenversorgung in den Ländern macht eine Übertragung auf die weltweite Situation schwierig.

\section{Fazit}

Die demografischen Daten in der SERENA-Studie entsprechen denen publizierter interventioneller Studien, es finden sich jedoch signifikante Unterschiede zu Krankheitscharakteristika bei Einschluss, insbesondere hinsichtlich der vorherigen Anwendung von Biologika. Insgesamt liefern daher Beobachtungsstudien wie diese wichtige Daten hinsichtlich der Verträglichkeit und Effektivität von Wirkstoffen unter Bedingungen der täglichen Praxis und damit wichtige Informationen über kontrollierte Studien hinaus.

Korrespondenz an:

Michael Sticherling, michael.sticherling@uk-erlangen.de 